\title{
ECLETICA
}

www.scielo.br/eq

Volume 30, número 4, 2005

\section{Síntese e estudo da fotoestabilidade de derivados azalactônicos encapsulados em $\boldsymbol{\beta}$-ciclodextrina.}

\author{
M. F. da Rosa*, F. C. Savariz \\ GIPeFEA - Grupo Interdisciplinar de Pesquisa em Fotoquímica e Eletroanalítica Ambiental \\ Química / CECE / Unioeste - CEP 85903-000, Toledo (PR), Brasil. \\ *mauriciorosa@unioeste.br
}

\begin{abstract}
RESUMO: Neste trabalho foram sintetizados 12 derivados azalactônicos pela metodologia clássica de Plöchl-Erlenmeyer. Destes, um foi avaliado quanto a fotoestabilidade em solução por meio de experimentos de fotodegradação utilizando agentes encapsulantes e diferentes solventes. Para a irradiação das amostras foi empregada uma lâmpada de mercúrio de $80 \mathrm{~W}$. Os resultados mostram que a fotodegradação do corante ocorre muito mais rapidamente em solventes polares e que o agente encapsulante é fundamental na sua fotoestabilidade.
\end{abstract}

Palavras-chave: azalactonas; fotodegradação; ciclodextrinas.

Introdução

As azalactonas são compostos heterocíclicos (Figura 1) conhecidos desde o século XIX e preparados pelo método clássico conhecido como síntese de Plochl-Erlenmeyer, em homenagem aos seus descobridores [1a-b].

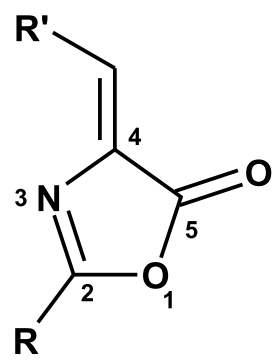

Figura 1 - Fórmula estrutural das azalactonas com o sistema de numeração.

O procedimento experimental mais comum de preparação de tais compostos é via reação entre um derivado $\mathrm{N}$-derivado da glicina (ou qualquer outro aminoácido) e um aldeído, utilizando anidrido acético como solvente e acetato de sódio como catalisador (Figura 2).

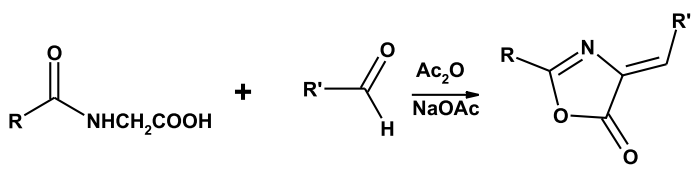

Figura 2 - Rota sintética das azalactonas

A aplicação de derivados azalactônicos é vasta, podendo ser utilizados como fluoróforos para o reconhecimento de proteínas e peptídeos [2], imobilizadores de enzimas [3], sensores fluorescentes de glicose [4], intermediários em síntese [5a-c], indicadores fluorescentes de $\mathrm{pH}$ em filmes poliméricos [6a] ou em matrizes sol-gel [6b], indicadores fluorescentes imobilizados em polímeros de íons $\mathrm{Fe}^{3+}$ [7], formação de complexos metálicos [8], complexos de transferência de carga [9a-b], geradores de segunda harmônico [10], entre outras.

Tendo em vista as propriedades emissivas destas moléculas, cogitou-se na utilização desta família de compostos como corantes para lasers. Corantes para lasers são moléculas orgânicas que, após serem eletronicamente excitadas, retornam ao estado fundamental emitindo radiação laser. A vantagem dos corantes sobre as outras fontes de 
emissão inclui a possibilidade de modulação do comprimento de onda da irradiação laser sob uma larga faixa espectral, um alto coeficiente de amplificação da luz emitida pelo meio ativo e a possibilidade de obter radiação em multibandas [11]. A aplicação de lasers de corantes na ciência é vasta, principalmente na medicina [12a-b]. Um corante produz emissão estimulada de luz (laser) na faixa espectral de sua fluorescência, logo para que um corante emita é necessário que ele seja promovido ao seu estado excitado por outra fonte de excitação. Desta forma é conveniente que o corante utilizado possua razoável fotoestabilidade, pois caso contrário a emissão cessará em curto espaço de tempo (photobleaching). Uma das formas de se alterar a reatividade de alguns compostos é trabalhar com suportes sólidos ou estruturas supramoleculares que possuam nanocavidades. Dentre estas as ciclodextrinas são as que mais se destacam, tanto pela sua acessibilidade quanto à facilidade de manuseio.

Ciclodextrinas (CD) são oligossacarídeos cíclicos que contêm $6(\alpha), 7(\beta)$ ou $8(\gamma)$ unidades de glicopiranose formando uma estrutura toroidal com ambas extremidades abertas e cujos diâmetros

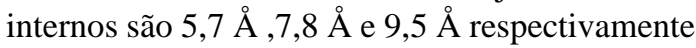
[13] (Figura 3).

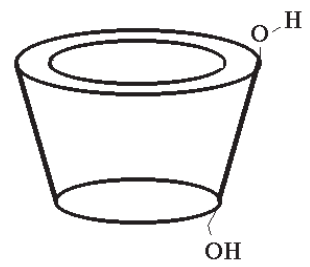

Figura 3 - Representação esquemática da estrutura da ciclodextrina

Esta característica estrutural permita a entrada de moléculas em seu interior através de uma interação hóspede-hospedeiro (guest-host) [13] e esta complexação, ou encapsulamento, apresenta uma variedade de aplicações industriais [14], principalmente nas áreas farmacêutica e de alimentos. Por exemplo, fármacos pouco solúveis em água podem ter sua solubilidade aumentada por este procedimento, uma vez que o interior da cavidade por ser hidrofóbica terá maior interação com o fármaco, e seu exterior de características hidrofílicas interagirá com a água. Além disto, alguns trabalhos mostram que o emprego de agentes encapsulantes aumenta a fotoestabilidade de compostos em solução [15a-c]. entretanto poucos exemplos foram encontrados na literatura sobre o emprego de complexos de inclusão $\operatorname{com} \beta$ $\mathrm{CD}$ como corantes para lasers [16a-c].

O objetivo deste trabalho é verificar a influência do solvente e de agentes encapsulantes na fotoestabilidade destes compostos, avaliandose a potencial utilização dos mesmos como corantes para lasers.

\section{Procedimento Experimental}

Os pontos de fusão foram determinados empregando-se um aparelho de ponto de fusão Fisatom 430D. Os espectros de absorção foram obtidos utilizando-se um espectrofotômetro Shimadzu UV-1601PC. Todos os solventes utilizados eram grau analítico e forma usados como recebidos. Como fonte de irradiação empregou-se uma lâmpada de mercúrio de $80 \mathrm{~W}$, conforme descrito em trabalho anterior [17]. Todas as amostras irradiadas tinham concentração de $5 \times 10^{-4}$ mol. $\mathrm{L}^{-1}$. Foi empregada a metodologia sintética descrita por Carter [18], baseada na síntese de Plöchl-Erlenmeyer apresentada a seguir:

1) Síntese do ácido hipúrico (AH) (Nbenzoilglicina) [19]

Em um erlenmeyer de $100 \mathrm{~mL}$ foram solubilizados $5 \mathrm{~g}$ de glicina (66,6 mmols) em $50 \mathrm{~mL}$ de $\mathrm{NaOH} 10 \%$. Em seguida foi adicionado em pequenas porções com agitação vigorosa $10,8 \mathrm{~g}$ (9,0 mL - 76,8 mmols) de cloreto de benzoíla. A solução resultante foi vertida em um béquer de 250 $\mathrm{mL}$, que já continha água e gelo, e foi adicionado gota a gota $\mathrm{HCl}$ concentrado até a indicação de $\mathrm{pH}$ $=2$ em papel indicador universal. $\mathrm{O}$ sólido obtido foi lavado com água fria em abundância, fervido com $\mathrm{CCl}_{4}$ para eliminação do ácido benzóico residual, filtrado e recristalizado em etanol:água (1:1). O rendimento da reação foi de $60 \%$ (PF: 186$189^{\circ} \mathrm{C}$-lit. $\left.187^{\circ} \mathrm{C}[19]\right)$.

2) Síntese da (4Z)-4-benzilideno-2-fenil1,3-oxazol-5-(4H)-ona (AZAO) 
Em um balão de $100 \mathrm{~mL}$ forma reunidos $1 \mathrm{~g}$ de AH (5,6 mmols), $1 \mathrm{~g}$ de acetato de sódio (17,2 mmols), $1 \mathrm{~mL}$ de benzaldeído (9,8 mmols) e $10 \mathrm{~mL}$ de anidrido acético (90,7 mmols). Esta mistura foi aquecida e agitada por 2 horas, mantendo-se a temperatura não superior a $80{ }^{\circ} \mathrm{C}$ neste período. Após o resfriamento da solução foram adicionados $20 \mathrm{~mL}$ de etanol e a mistura foi deixada sob refrigeração (freezer) por 12 horas. O sólido precipitado foi filtrado e lavado com água e etanol gelado. O produto obtido foi recristalizado em tolueno, obtendo-se cristais em forma de agulhas de coloração amarela pálida. O rendimento da reação foi de $35 \%$ (PF: $164-167^{\circ} \mathrm{C}-$ lit. $165^{\circ} \mathrm{C}[10]$ ).

Este foi o procedimento geral empregado na síntese de todos os outros derivados azalactônicos.

3) Preparação das amostras para irradiação sem $\beta$-CD

Prepara-se inicialmente $25 \mathrm{~mL}$ de uma solução-mãe de AZA2 em clorofórmio de concentração $5 \times 10^{-3} \mathrm{~mol} \mathrm{~L}^{-1}$. Desta solução retiramse alíquotas de $0,1 \mathrm{~mL}$ que são transferidas para balões volumétricos de $10 \mathrm{~mL}$. Deixa-se o solvente evaporar e então completa-se o volume com o solvente adequado. A solução é submetida ao ultrassom por 15 minutos para completa solubilização do sólido e a eliminação do oxigênio da amostra.

4) Preparação das amostras para irradiação com $\beta$ - $C D$

Prepara-se $25 \mathrm{~mL}$ de uma solução $5 \times 10^{-3}$ mol L ${ }^{-1}$ de $\beta$-CD $(0,14185 \mathrm{~g})$ em acetona ou clorofórmio. Desta solução retira-se uma alíquota de 1,0 mL e transfere-se para um balão volumétrico de $10 \mathrm{~mL}$. Transfere-se para o mesmo balão uma alíquota de $0,1 \mathrm{~mL}$ de AZA2 em clorofórmio. Agitase a solução resultante e deixa-se o solvente evaporar. Completa-se o volume com isopropanol e submete-se a solução ao ultrassom. Este procedimento foi feito somente com isopropanol devido a insolubilidade da $\beta$-CD em hexano.

\begin{tabular}{|c|c|c|c|c|}
\hline & AZA & $\mathrm{R}^{\prime}$ & $\mathrm{PF}\left({ }^{\circ} \mathrm{C}\right)$ & PF $\left({ }^{\circ} \mathrm{C}\right)[$ lit. $]$ \\
\hline & 0 & fenil & $164-7$ & $165-6[10]$ \\
\hline & 1 & 4-hidroxifenil & $175-9 *$ & $144[20]$ \\
\hline & 2 & 4-(N,N-dimetilamino)fenil & $210-2$ & $214-5[20]$ \\
\hline & 3 & 2-furil & $168-70$ & $173[21]$ \\
\hline & 4 & 2-hidroxifenil & $134-6 *$ & - \\
\hline & 5 & 3,4-dimetoxifenil & $149-50$ & $151-2[22]$ \\
\hline & 6 & 2-indolil & $200-2^{*}$ & - \\
\hline & 7 & 2-hidroxi-3,5-di-t-butilfenil & $55-7$ & - \\
\hline & 8 & 4-nitrofenil & $240-2$ & $246-7$ [10] \\
\hline & 9 & 4-fluorofenil & $174-6$ & $176-7[10]$ \\
\hline \multirow[b]{2}{*}{ * derivados acetilados } & 10 & 4-metoxifenil & $155-9$ & $159-61[10]$ \\
\hline & 11 & 3-hidroxi-4-metoxifenil & $155-8$ & 158 [23] \\
\hline
\end{tabular}

Tabela 1 - Derivados azalactônicos sintetizados. 


\section{Resultados e discussão}

Até o momento foram sintetizados 13 derivados azalactônicos. Todos foram purificados por recristalizações sucessivas e caracterizados por ponto de fusão e espectros de infravermelho (Tabela 1).

Por apresentar o maior número de citações na literatura $[4,6,7,24]$ o derivado identificado como AZA2 [(4Z)-4-[4-( $N, N$-dimetilamino)-benzilideno]-2fenil-1,3-oxazol-5-(4H)-ona] foi escolhido para este estudo inicial. Para se determinar a fotoestabilidade deste composto em solução, e verificar a influência do solvente no processo fotodegradativo, este derivado foi irradiado em solução de hexano ou isopropanol, utilizando-se uma lâmpada de mercúrio de $80 \mathrm{~W}$.

A fotodegradação da AZA2 foi acompanhada pelos respectivos espectros de absroção em função do tempo de irradiação. Verificase que em solventes apolares (Figura 4) esta reação ocorre muito mais lentamente do que em solventes polares (Figura 5). Na Figura 5, observa-se ainda a presença de um ponto isosbéstico em torno de 270 nm.

Considerando-se uma cinética de pseudoprimeira ordem, o gráfico de $\ln \left(\mathrm{I}_{0} / \mathrm{I}\right)$ vs tempo deve apresentar uma dependência linear e o coeficiente angular da reta fornece a constante de velocidade aparente $\left(\mathrm{k}_{\mathrm{ap}}\right)$ do processo. Para a amostra em hexano verifica-se um ótimo ajuste linear $\left(R^{2}=0,995\right)$, cujo coeficiente angular fornece $\mathrm{k}_{\mathrm{ap}}=8 \times 10^{-4} \mathrm{~L} \mathrm{~mol}^{-1}$ $\min ^{-1}$ (Figura 6A). A Figura 6B mostra o mesmo procedimento para a amostra em isopropanol e

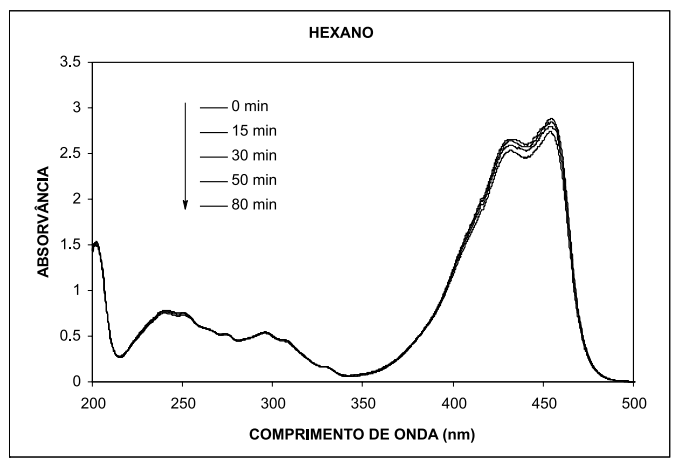

Figura 4 - Espectros de absorvância em função do tempo de irradiação da AZA2 em hexano $\left(5 \times 10^{-5} \mathrm{~mol} \mathrm{~L}^{-1}\right)$.

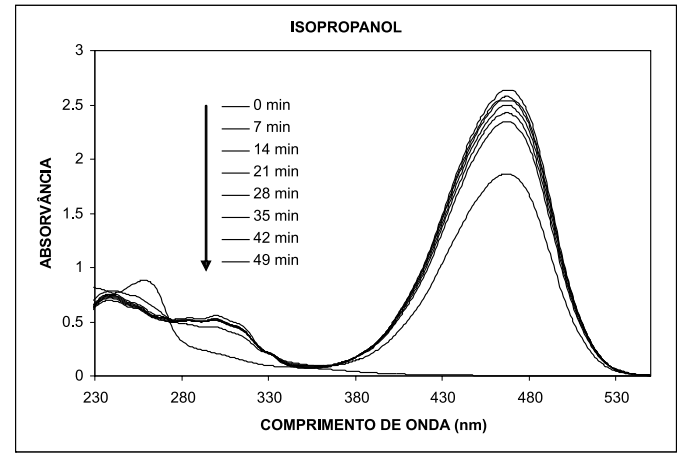

Figura 5 - Espectros de absorvância em função do tempo de irradiação da AZA2 em isopropanol $\left(5 \times 10^{-5} \mathrm{~mol} \mathrm{~L}^{-1}\right)$.

observa-se que, para os 35 minutos iniciais do experimento, a amostra apresenta razoável dependência linear, apesar do ajuste obtido não ter sido tão bom quanto o anterior $\left(\mathrm{R}^{2}=0,9702\right)$. $\mathrm{O}$ coeficiente angular da melhor reta para este intervalo é $3 \times 10^{-3}$, sendo este então considerado o valor de $\mathrm{k}_{\mathrm{ap}}\left(\mathrm{L} \mathrm{mol}^{-1} \mathrm{~min}^{-1}\right)$.

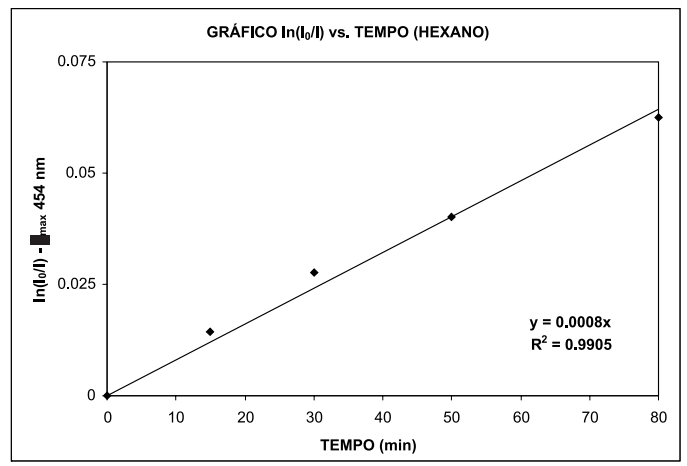

Figura 6A - Gráfico da absorvância normalizada $\ln \left(\mathrm{I}_{0} / \mathrm{I}\right)$ vs tempo para AZA2 em hexano.

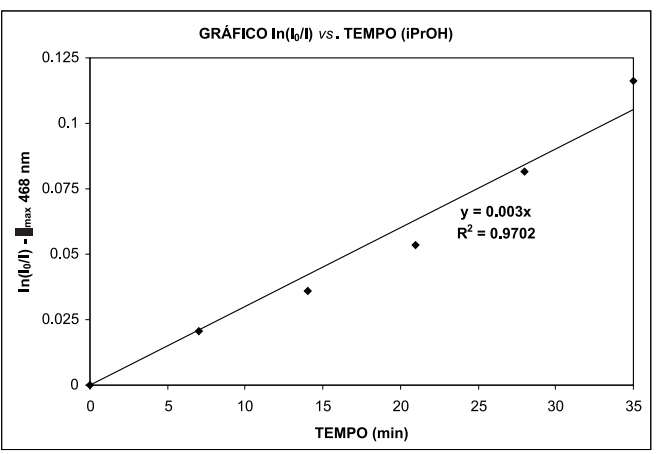

Figura 6B - Gráfico da absorvância normalizada $\ln \left(\mathrm{I}_{0} / \mathrm{I}\right)$ vs tempo para AZA2 em isopropanol. 
Considerando-se agora que a fotodegradação da AZA2 em isopropanol ocorre segundo uma cinética de segunda ordem (d[AZA2]/ $\mathrm{dt}=-\mathrm{k}[\mathrm{AZA} 2]^{2}$ ) e fazendo-se o gráfico $1 / \mathrm{I} v \mathrm{~s}$ tempo, verifica-se que o ajuste não é muito diferente do obtido para a cinética de primeira ordem (Figura 7A). Colocando-se no gráfico todos os pontos experimentais obtém-se um excelente ajuste da curva para um comportamento exponencial (Figura 7B), entretanto neste observa-se que o erro do valor pré-exponencial (A) é maior do que o valor do parâmetro empregado para o ajuste.

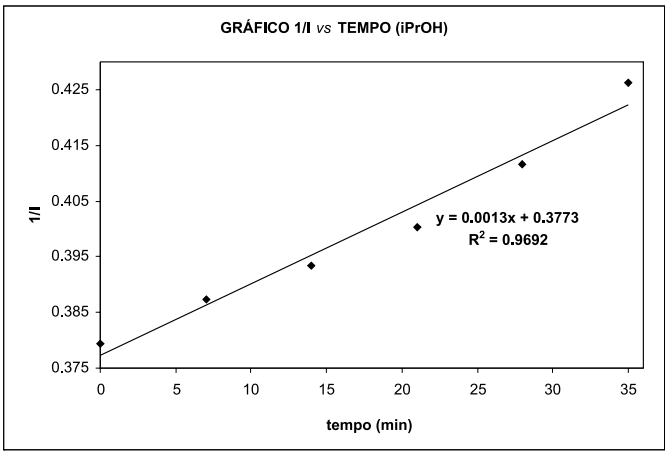

Figura 7A - Gráfico do recíproco da absorvância (1/I) vs tempo para AZA2 em isopropanol.

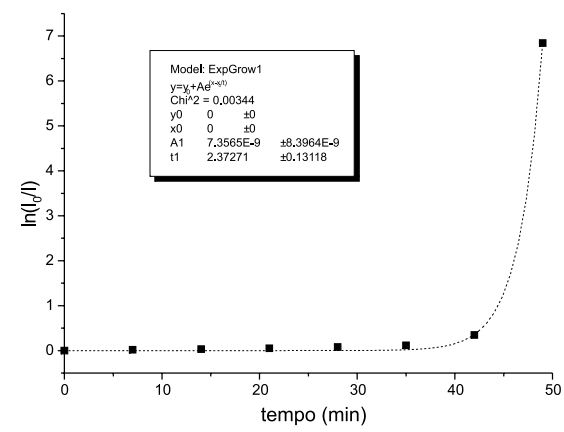

Figura 7B - Ajuste exponencial do gráfico da absorvância normalizada $\left(\ln \left(\mathrm{I}_{0} / \mathrm{I}\right)\right)$ vs tempo para AZA2 em isopropanol.

Desta forma fica evidente que a cinética de fotodegradação da AZA2 em isopropanol não segue uma cinética simples, cabendo maiores investigações sobre o processo.

Para se verificar a influência de agentes encapsulantes sobre o comportamento fotoquímico do corante, repetiu-se o experimento anterior adicionando-se $\beta$-CD à solução. Na Figura 8 é apresentado o espectro de absorção em função do tempo de irradiação da AZA2 em isopropanol com emprego de $\beta$-CD. Observa-se claramente que a presença de $\beta-C D$ aumenta consideravelmente o tempo de vida do corante submetido a irradiação. Pelo coeficiente angular do gráfico $\ln \left(\mathrm{I}_{0} / \mathrm{I}\right)$ vs tempo (Figura 9) para o experimento com $\beta$-CD determinouse a constante de velocidade da reação como sendo $\mathrm{k}=4 \times 10^{-4} \mathrm{~L} \mathrm{~mol}^{-1} \mathrm{~min}^{-1}$, ou seja, um valor dez vezes menor do que o calculado para o experimento sem $\beta-\mathrm{CD}$.

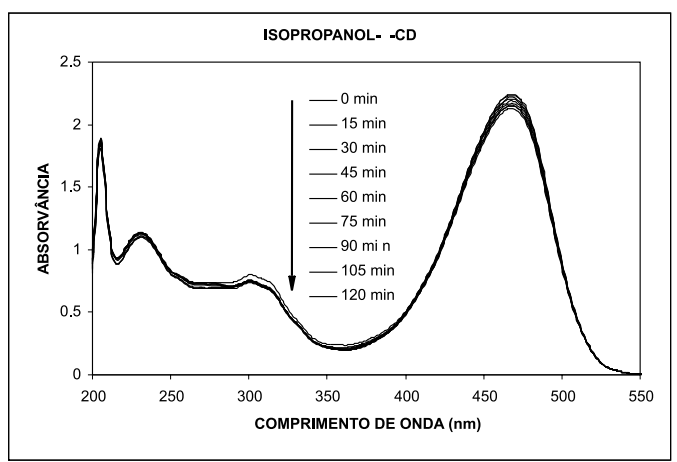

Figura 8 - Espectros de absorção da AZA2 em isopropanol com adição de $\beta$-CD.

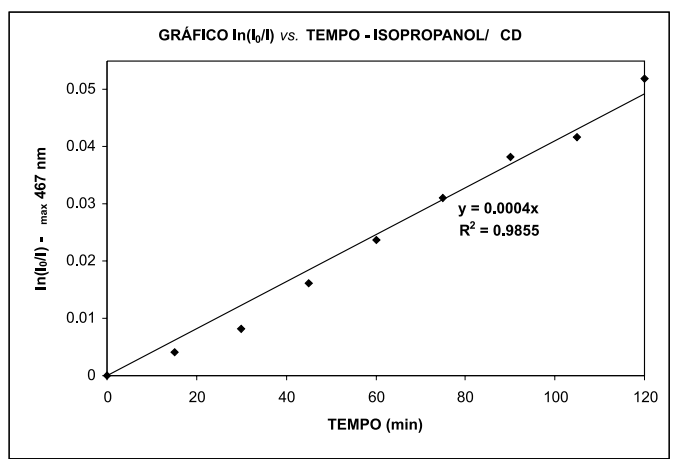

Figura 9 - Gráfico da absorvância normalizada $\ln \left(\mathrm{I}_{0} / \mathrm{I}\right)$ vs tempo para AZA2 em isopropanol com $\beta$-CD. 
Em função dos resultados apresentados podemos concluir que o solvente afeta a velocidade de degradação do corante submetido à irradiação. Uma hipótese para a degradação mais rápida em isopropanol está relacionada a uma possível transferência de hidrogênio do solvente para o soluto no estado excitado [25]. Esta hipótese é favorecida quando verifica-se o comportamento fotoquímico na presença de agentes encapsulantes, pois neste caso observa-se maior estabilidade do corante frente a irradiação. Isto ocorre porque, uma vez encapsulado, o soluto tem pouco contato com o solvente, sendo, portanto, a transferência hidrogênio minimizada. Claramente estes não são os únicos processos atuando no sistema, pois de outra forma não seria observado a fotodegradação do corante em solução com solvente apolar. Como a faixa espectral da lâmpada utilizada no experimento é larga, tendo também emissão na região do ultravioleta, radiações desta ordem são suficientemente energéticas para quebrar ligações e provocar a degradação do corante.

\section{Conclusões}

Dos doze derivados azalactônicos sintetizados um deles foi estudado quanto ao seu comportamento fotoquímico frente a irradiação com uma lâmpada de mercúrio de $80 \mathrm{~W}$. Determinou-se que a constante de velocidade para o processo fotodegradativo em solvente apolar (hexano) era de $8 \times 10^{-4} \mathrm{~L} \mathrm{~mol}^{-1} \mathrm{~min}^{-1}$, enquanto que em solvente polar prótico (isopropanol) esta era de $3 \times 10^{-3} \mathrm{~L} \mathrm{~mol}^{-1}$ $\min ^{-1}$, evidenciando a influencia do solvente no mecanismo de fotodegradação. A presença de agentes encapsulantes reduz esta constante de velocidade para $4 \times 10^{-4} \mathrm{~L} \mathrm{~mol}^{-1} \mathrm{~min}^{-1}$, mostrando que tais agentes podem contribuir significativamente para ao maior tempo de vida destes compostos quando submetidos a irradiação contínua. Um estudo mais detalhado sobre os fotoprodutos formados está sendo realizado.

\section{Agradecimentos}

Ao Fundo Paraná convênio 04/01. A Leonice Bezerra e ao DQI/IQ/UFRJ pelos espectros de FT-IR.

Recebido em: 08/07/2005

Aceito em: 16/09/2005

M. F. da Rosa, F. C. Savariz. Synthesis and photostability studies of azlactone derivatives encapsulated in b-cyclodextrin.

ABSTRACT: In this work 12 azlactone derivatives were synthetized using the classical Plöchl-Erlenmeyer methodology. One of them was evaluated in respect of its photostability in solution using solvents with different polarities and with addition of encapsulating agents. The photodegradation experiments were done using a $80 \mathrm{~W}$ mercury lamp as source of light. The results show faster dye degradation in polar media, but when encapsulating agents is present this photodegradation is lowered.

Key words: azlactones; photodegradation; cyclodextrin.

\section{Referências}

[1]a) A. Plöchl, Ber. 16, (1883) 2815; b) E. Erlenmeyer, Ann. 275 (1893) 3.

[2] G. Kóczán, G. Csík, A. Csámpai, E. Balog, S. Bosze, P. Sohár, F. Hudecz., Tetrah. 57 (2001) 4589.

[3] S. M. Heilmann, G. J. Drtina, L. C. Haddad, J. K. Rasmussen, B. N. Gaddam, J. J. Liu, R. T. Fitzsimons, D. D. Fansler, J. R. Vyvyan, Y. N. Yang, T. J. Beauchamp, J. Mol. Cat. B 30(1) (2004) 33.

[4] K. Ertekin, S. Cinar, T. Aydemir, S. Alp, Dyes \& Pigm. 67 (2005) 133. [5]a) R. Huisgen H. Gotthardt, H. O. Bayer, Angew. Chem. Int. Engl. Ed. 3(2) (1964) 135; b) M. Hashimoto, M. Sugiura, S. Terashima., Tetrah. 59 (2003) 3063; c) H. Joshi, P. Upadhyay, D. Karia, A. J. Baxi, Eur. J. Med. Chem. 38(9) (2003) 837.

[6] a) K. Ertekin, S. Alp, C. Karpire, B. Yenigül, E. Henden, S. Içli., J. Photochem. Photobiol. A 137 (2000) 155; b) K. Ertekin, C. Karapire, S. Alp, B. Yenigül, S. Içli, Dyes \& Pigm. 56(2) (2003) 125.

[7] G. Ozturk, S. Alp, K. Ertekin, Dyes \& Pigm. (2005), 
no prelo (artigo acessado no sítio www.sciencedirect.com em 25 de novembro de 2005).

[8] I. S. Ahmed \& E. H. El-Mossalamy, J. Anal. Appl. Pyrolysis 70(2) (2003) 679.

[9]a) E. H. El-Mossalamy , A. S. Amin, Microchem. J., 52 (1995) 263; b) E. H. El-Mossalamy, A. S. Amin, A. A. Khalil, Spectrochim. Acta A 58 (2002) 67.

[10] M. Kitazawa, R. Higuchi, M. Takahashi, T. Wada, H. Sasabe, J. Phys. Chem. 99 (1995) 14784.

[11] B. M. Uzhinov \& S. I. Druzhinin, Rus. Chem. Rev. 67(3) (1998) 123.

[12]a) S. R. Tan \& W. D. Tope, J. Am. Acad. Dermat. 51(4) (2004) 592; b) S. R. Tan, A. Bialostocki, J. R. Armstrong, Brit. J. Plastic Surg. 57(4) (2004) 303; J.-L. Michel, C. Grognard, G. Toubel, H. Cartier, T. Fusade, D. Perrin, Med. Laser Apll. 19(3) (2004) 136.

[13] J. Szejltli, Chem Rev. 98 (1998) 1743.

[14] A. R. Hedges, Chem. Rev. 98 (1998) 2035.

[15]a) R. Guether \& M. V. Reddington, Tetrah. Lett 38(35) (1997) 6167; b) J. Mielcarek \& E. Daczkowska J. Pharm.Biomed. Anal. 21(2) (1999) 393; c) M. A. Bayomi, K. A. Abanumay, A. A. Al-Angary, Int. J. Pharm. 243(1-2) (2002) 107; d) H. H. Tønnesen, M. Másson, T. Loftsson, Int. J. Pharm. 244(1-2) (2002) 127; e) R. Pomponio, R. Gotti, J. Fiori, V. Cavrini , P. Mura, M. Cirri, F. Maestrelli, J. Pharm. Biomed. Anal. 35(2) (2004) 267. [16]a) I. R. Politzer, K. T. Crago, T. Hampton, J. Joseph, J. H. Boyer, M. Shah, Chem. Phys. Lett. 159(2-3) (1989) 258 ; b) Y. Degani \& I. W. Y. Haas, Chem Phys. Lett. 104(5), (1984) 496; c) V. K. Indirapriyadharshini, P. Karunanithi, P. Ramamurthy, Langmuir 17(13) (2001) 4056.

[17] R. A. Bariccatti, M. F. Rosa, J. D. S. Oliveira, Eclet. Quim. 29(2) (2004) 15.

[18] H. E. Carter, Org. React. 3 (1946) 198.

[19] A. I. Vogel, Química Orgânica Qualitativa vol.2, Ao Livro Técnico, Rio de Janeiro, $2^{a}$ edição, 1973. p.619.

[20] S. Içli, H. Icil, S. Alp, H. Koc, A. McKillop, Spectrosc. Lett. 27 (1994) 1115.

[21] B. M. Krasovitskii, A. V. Yudashkin, I. V. Lysova, L. S. Afanasiadi, A. E. Lipkin, Khim Geterotsikl Soedin. (Eng. Transl.) (12) (1979) 1303.

[22] J. S. Buck \& W. S. Ide, Org. Synth. 2 (1943) 55.

[23] P. Kumar, H. D. Mishra, A. K. Mukerjee, Synthesis (1980) 836.

[24] K. Ertekin; S. Alp; I. Yalcin, Dyes \& Pigm., 65(1) (2005) 33.

[25] N. J. Turro, Modern Molecular Photochemistry, $1^{\text {a }}$ edição. Mill Valley: University Science Books, 1991. pp. 362. 